\title{
The experience of a multidisciplinary uro- oncological team in a provincial hospital in Cuba
}

\begin{abstract}
Objective: The objective of the present article is to advertise the experience of a multidisciplinary urologic oncology team in a provincial hospital in Cuba.

Methods: Description and presentation of experience and working algorithm followed by three urologists, an oncologists and a trained nurse in a provincial general hospital from 1993 until now, as well as the interaction with other disciplines related to the uro-oncological treatment.

Results: The medical care, teaching, and research aspects have provided us with a greater understanding of the oncological disease in the area of urology.

Conclusion: The integration of an uro-oncological branch in a provincial general hospital is possible whenever exists enough will in the hospital administration and the readiness of a multidisciplinary medical staff (urologists, oncologists, radiotherapists, etc.) to collaborate and to put work in the creation and implementation of the same. The result will be an interdisciplinary and qualified care of patients with uro-oncological diseases.
\end{abstract}

Keywords: uro-oncology, multidisciplinary team, working algorithm, integration
Volume 7 Issue I - 2019

\author{
Jorge Luis Sagué Larrea,' Luis Armas \\ Martínez, ${ }^{2}$ Zulma Doimeadios Rodríguez, ${ }^{3}$ \\ Oricel Estrella Fernández Oliva, ${ }^{4}$ \\ 'Urology, Espoch Chimborazo, Ecuador \\ 2Urology, less Babahoyo, Ecuador \\ ${ }^{3}$ Medical Doctor, Espoch Chimborazo, Ecuador \\ ${ }^{4}$ Medical Doctor, less Babahoyo, Ecuador
}

Correspondence: Jorge Luis Sagué Larrea, Espoch

Chimborazo, Uroncology professor, Street Antonio Salas and Samaniego, Riobamba, Chimborazo, Ecuador, Tel 097908215I, Email saguej@yahoo.com

Received: January 29, 2017 | Published: February 28, 2019

\section{Introduction}

The care of an oncological patient requires the interaction of different medical and other health care disciplines. ${ }^{1-4}$ This makes necessary the integration, collaboration and coordination of health care professionals from different disciplines, which offer in a professional manner their specialized knowledge for the well-being of the patient. Theoretically this interaction should lead to a benefit for the patient. A multidisciplinary uro-oncological team was formed in our hospital with the objective to strengthen and develop medical care in this discipline. ${ }^{5,4}$ This task was not easy to manage, so we decided to reveal the experience we acquired throughout several years of hard work.

\section{Materials and methods}

In January 1993 was already found the uro-oncological section as a part of the oncological department of our hospital. It was made up of three urologists, an oncologist and a nurse, who were dedicated full time to this activity. This team was in charge of establishing work relationships between the different specialized disciplines which belong to the area of oncology. The hospital administration assigned an eight- bed male room, and if necessary according to the demand access to the beds of the general women's warded as well as to the section of radiation treatment. The work algorithm is shown on Figure 1.

\section{Results and discussion}

\section{Medical care}

Doctor's consultations take place weekly, sometimes with special focus, according to the incidence of some diseases in our environment (prostate and bladder). In the consultation of a vesical neoplasia, will be employed a diagnostic procedure with cysto scopies and biopsies if necessary and the post operatory follow up of the patients. In the prostatic cancer consultation we perform biopsies and FNAB's as well as post surgical and radiation follow-up's. ${ }^{6-8}$ The endo vesical or systemic chemotherapy is given in the chemotherapy section ${ }^{9}$ by a well-trained nurse and under the supervision of one of the specialized doctors of the team. In the general consultations, there will be seen the broad range of uro -oncologic pathologies, for example renal diseases or pathologies of the genitals. The patients which present themselves for an uro-oncologic consultation, generally have been studied by the urologists of the hospital ${ }^{10}$ or from municipal hospitals in the province. We have a weekly surgical shift for the treatment of uro-oncological diseases and when we do not complete it with enough surgeries, we include cases of the general urology service, which is almost always overcrowded. Sometimes when we have to perform a major surgery bladder replacement, radical prostatectomy, etc. - the administration provides us with an extra operating session in order to not interrupt the normal flow of the planned operations. In the surgical interventions (Table 1) are participating always specialists and residents of the general surgery service, as well as the oncologist. ${ }^{11-14}$ from our team. The planning of the development of these activities is made by the heads of the Urology and Oncology departments. Pre-operative case discussions together with the urologic team are carried out weekly to achieve a consensus. In case of a deeply rooted doctor-patient relationship or another personal interest of any kind on the part of the doctor or the patient, we favor the same doctor to perform the surgery with the support of our team.

\section{Teaching}

The rotation of medical students through the oncologic service is not scheduled in Cuba, but in the urologic service the students are rotating into the uro-oncological section so that both, the urologist and oncologist maintain their teaching lessons and training with the students in order to deepen their knowledge. When the fifth-year medical students complete their rotation, free-choice classes on specific pathologies are offered (lasting twenty one days). During their residency in the oncologic and neurologic departments, the doctors have periods of rotation through these disciplines, with frequently 
performed evaluations. We also offer training and post-graduate courses in uro-oncological diseases for urologists, oncologists and related specialists. ${ }^{5,16}$, including for the personnel from nearby provinces.

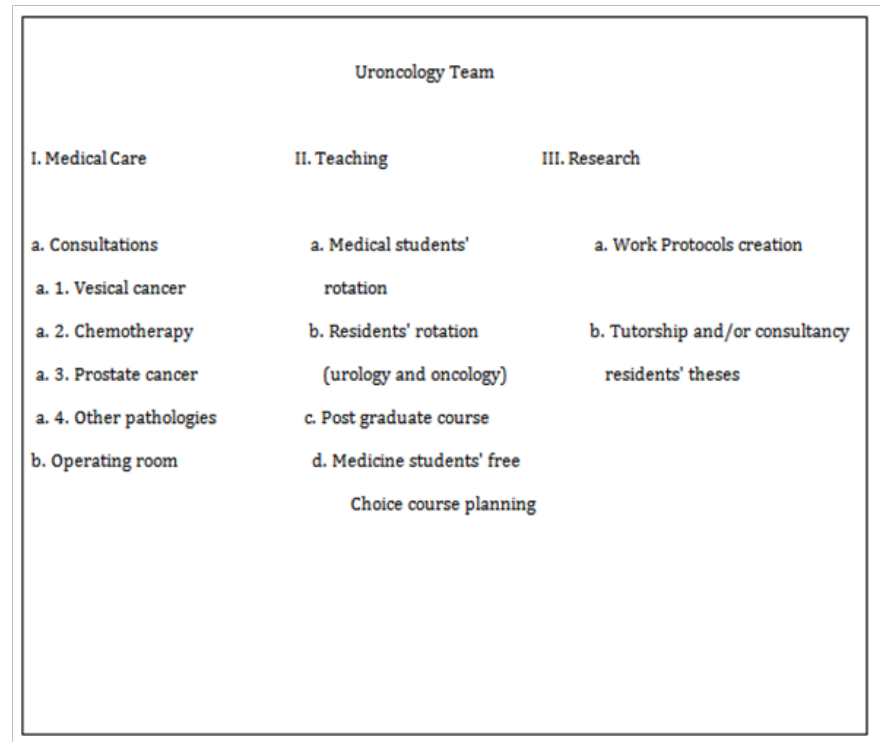

Figure I Work algorhythm.

Source: Holguín Teaching General Hospital Uroncological Section.

Table I Uroncology Group, Results of the work during the period 2012-20I6

\begin{tabular}{|c|c|c|}
\hline I. Mayor surgical Interventions & No. & $\%$ \\
\hline Radical prostatectomy & 120 & 14,3 \\
\hline Radical cystectomy & 61 & 7,3 \\
\hline RTU bladder cáncer & 305 & 36,4 \\
\hline Partial cystectomy & 118 & 14,1 \\
\hline Alloplastic bladder substitution & 23 & 2,7 \\
\hline Radical nephrectomy & 72 & 8,6 \\
\hline Partial nephrectomy & 53 & 6,3 \\
\hline Nephrourecterectomy & 9 & 4,7 \\
\hline Penile amputation & 16 & 1,9 \\
\hline Partial amputation of the penis & 31 & 3,7 \\
\hline Subtotal & 838 & 100,0 \\
\hline \multicolumn{3}{|l|}{ II. Teaching } \\
\hline Rotations of medical students & 50 & \\
\hline Residents who rotated & 23 & \\
\hline Postgraduate courses and trining performed & 17 & \\
\hline \multicolumn{3}{|l|}{ III. Research. } \\
\hline Creating Service Work Guides & 1 & \\
\hline Creating Research Protocols & 6 & \\
\hline Resident Thesis Tutoring & 19 & \\
\hline
\end{tabular}

Source: Holguín Teaching General Hospital Uroncological Section.

\section{Research}

Several working protocols for research were elaborated, which have to be executed by the urologic and oncologic service staff members. These protocols are not mandatory but contribute to unify criteria of different disciplines (surgery, radiotherapy, immunotherapy, etc.). ${ }^{17}$ Before forming this team the urologists of our hospital did not formally follow work protocols and did their work according to their own experience (empirically or scientifically), meanwhile the oncologists for their part were focused on radiation treatment. Since the creation of our multidisciplinary team, each one of the disciplines have had to give up their egoistic behavior of playing the main role in the process of the treatment of the disease. Naturally the interpersonal relationship is a very important factor to create and apply with success a single therapeutic guideline. Before the planning of any research project there is the relationship with other disciplines. The resident doctors who are finishing their residency in the oncological area of urology and prepare their final thesis can count on the tutorship and guidance of the multidisciplinary uro-oncological team members. All the team members should be highly motivated, abandon their selfish behavior and adopt a more cooperative conduct, as it is generally pursued by different multidisciplinary teams worldwide.

\section{Conclusion}

The integration of an uro-oncological multidisciplinary team in a provincial general hospital is possible whenever there exists enough will on the part of the hospital administration and the will of specialized physicians (urologists, oncologists, radiotherapists, etc.) from different disciplines to collaborate in a multidisciplinary team to establish a multi factorial care of oncological patients in the genitourinary field.

\section{Acknowledgments \\ None.}

\section{Conflicts of interest}

The authors declared there is no conflict of interest.

\section{References}

1. Christoph Heintze, Dorothea Matysiak-Klose, Antje Howorka, et al. Statements of general practitioners on cooperation with specialists and a future health care system. Medizinische Klinik. 2004;99(8):430-434.

2. Volkmer BG, Franziska Stredele RJ, Petervari M, et al. Veränderungen des G- DRG-Systems für die Urologie im Jahre 2012. Der Urologe. 2012;51(8):1109-1116.

3. Ruhstaller $\mathrm{T}$, Roe H, Thurlimann B, et al. The multidisciplinary meeting:an indispensable aid to communication between different specialities. Eur J Cancer. 2006;42(15):2459-2462.

4. Beermann S, Weißbach L. Interdisciplinary treatment of urological tumors: a mixed-method study. Der Urologe. 2012;51(8):1085-1088.

5. Grimm MO, Winkler I, Fetter I, et al. Renaissance of immuno-oncology for urological tumors Current status. Urologe A. 2016;55(5):621-626.

6. Sandra Beermann, Denny Chakkalakal, Rebecca Muckelbauer, et al. "We talk it over"- mixed-method study of interdisciplinary collaborations in private practice among urologists and oncologists in Germany. BMC Cancer. 2014;14(1):746.

7. Sevilla Paz Soldán, Ricardo. Importancia de un equipo multidisciplinario en tratamiento y manejo de enfermedades desde edades tempranas de la vida. Gac méd Boliv. 2013;36(2):118-118. 
8. Aizer AA, Jonathan Paly, Efstathiou JA. Multidisciplinary Care and Management Selection in Prostate Cancer. Seminars in radiation oncology. 2013;23(3):157-64.

9. Grimm MO, Winkler I, Fetter I, et al. Renaissance of immuno-oncology for urological tumors Current status. Urologe A. 2016;55(5):621-626.

10. Clarke CA, Glaser AL, Leung R, et al. Prevalence and characteristics of cancer patients receiving care from single vs. multiple institutions. Cancer Epidemiol. 2017;46(4):27-33.

11. Wright FC, Lookhong N, Urbach D, et al. Multidisciplinary cance conferences: identifying opportunities topromote implementation. Ann Surg Oncol2009;(10):2731-2737.

12. Katri Huutoniemi, Julie Thompson Klein, Henrik Bruun, et al. Analyzing interdisciplinarity: Typology and indicators Original Research Article. Research Policy. 2010;39(1):79-88.

13. Jalil R, Ahmed M, Green JS, et al. Factors that can make an impact ondecision-making and decision implementation in cancer multidisciplinary teams: an interview study of the provider perspective. Int J Surg. 2013,11(5):389-339.

14. Prades Joan, Borrás Josep. Multidisciplinary cáncer care in Spain, or when the function creates the organ: qualitative interview study. $B M C$ Public Health. 2011;11(1):141.

15. Sevilla Paz Soldán, Ricardo. Importancia de un equipo multidisciplinario en tratamiento y manejo de enfermedades desde edades tempranas de la vida. Gac méd Boliv. 2013;36(2):118-118.

16. El Khoury, Mario Chahrouri, Hachem C, et al. Evaluation of multidisciplinary team meetings in uro-oncology. The Lebanese medical journal. 2016;64(2):84-90.

17. Prostate Cancer Unit Initiative in Europe: A position paper by the European School of Oncology. Crit Rev Oncol Hematol. 2015;95(2):133-143. 